\section{$\underset{\substack{\text { hommes } \\ \text { \& migrations }}}{ }$}

\section{Hommes \& migrations}

Revue française de référence sur les dynamiques

migratoires

$1324 \mid 2019$

Religion et discrimination

\title{
De la lutte contre les discriminations à la promotion de la laïcité
}

\section{Edwin Hatton}

\section{(2) OpenEdition \\ Journals}

Édition électronique

URL : https://journals.openedition.org/hommesmigrations/8304

DOI : 10.4000/hommesmigrations.8304

ISSN : 2262-3353

Éditeur

Musée national de l'histoire de l'immigration

Édition imprimée

Date de publication : 1 janvier 2019

Pagination : 49-55

ISBN : 978-2-919040-44-5

ISSN : $1142-852 X$

Référence électronique

Edwin Hatton, " De la lutte contre les discriminations à la promotion de la laïcité », Hommes \& migrations [En ligne], 1324 | 2019, mis en ligne le 01 janvier 2022, consulté le 08 janvier 2022. URL : http://journals.openedition.org/hommesmigrations/8304 ; DOI : https://doi.org/10.4000/ hommesmigrations.8304 


\section{De la lutte contre}

les discriminations

à la promotion

de la laïcité

Edwin Hatton,

consultant à Coopaname.

Si l'appartenance religieuse constitue bien l'un des motifs de discrimination punis par la loi, son traitement politique est devenu problématique en France. Depuis 2012, la question du fait religieux dans l'espace public donne prise à une politisation croissante de la laïcité.

Ainsi, l'action du pouvoir exécutif s'est progressivement orientée vers la promotion, voire

la défense, de la laïcité. Cet article montre comment cette situation a eu tendance à marginaliser la lutte contre les discriminations. Or ce glissement qui confère à la religion une visibilité accrue est paradoxal dans un pays où deux-tiers des personnes se déclarent athées ou sans religion.

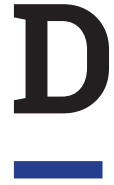

epuis une quinzaine d'années, les discriminations et la gestion du fait religieux se sont imposés comme des problèmes publics. Une forte porosité caractérise ces deux sujets qui renvoient tous deux au rapport complexe qu'entretient la France avec ses minorités (ethniques, religieuses et autres). Cette proximité se retrouve dans la façon dont ces thématiques sont traitées par les acteurs publics. Ainsi, dans les collectivités territoriales, les professionnels en charge de la lutte contre les discriminations se sont saisis en premier de la question du fait religieux à la fin des années 2000. À partir de 2012, les politiques de promotion de la laïcité se sont développées tandis que la lutte contre les discriminations a commencé à décliner. Ce processus s'est accentué après les attentats de 2015 qui ont entrainé une mobilisation sans précédent des pouvoirs publics en faveur de la laïcité tout en confirmant la marginalisation de la lutte contre les discriminations. Cet article entend retracer les grandes étapes de ce glissement et montrer l'articulation entre ces deux causes, en particulier dans les politiques territoriales. Il s'appuie sur des expériences professionnelles dans le champ de la lutte contre les discriminations ${ }^{1}$ et sur un

1. J'ai été consultant à Altidem (2007-2009), puis chef de projet Lutte contre les discriminations à la mairie de Grenoble (2010-2013) avant de devenir consultant-formateur indépendant en 2014. 
mémoire universitaire ${ }^{2}$ réalisé en 2017 sur la promotion de la laïcité par les collectivités territoriales ${ }^{3}$.

\section{L'invention de la discrimination et la «nouvelle laïcité »}

L'histoire de la lutte contre les discriminations en France est connue 4 . Après une phase de déni, l'État français reconnaît en 1998 les discriminations raciales à l'emploi comme un problème public. Sous l'influence de l'Union européenne, il se dote d'un arsenal juridique et politique destiné à lutter contre les discriminations: loi du 16 novembre 2001, mise en place de commissions départementales - Commission départementale d'accès à la citoyenneté (Codac), puis Commission pour la promotion de l'égalité des chances et la citoyenneté (Copec), création d'un organisme d'aide aux victimes de discrimination - Groupe d'étude et de lutte contre les discriminations (Geld), puis Haute Autorité de lutte contre les discriminations et pour l'égalité (Halde) -, lancement des premiers plans territoriaux de lutte contre les discriminations et des projets européens «Equal»... Tout ceci contribue à la formation d'un nouveau référentiel d'action publique qui rompt avec l'antiracisme (on ne lutte plus contre une idéologie mais contre des pratiques) et l'intégration (plutôt que d'aider les populations à s'intégrer, on s'attaque aux mécanismes qui les excluent). Symbole de ce changement de paradigme, le Fonds d'action sociale pour les travailleurs immigrés et leur famille (Fastif) devient le Fonds d'action sociale, d'intégration et de lutte contre les discriminations (Fasild) en 2001.

\section{(l. Après une phase de déni, l'État français reconnaît en 1998 les discriminations raciales à l'emploi comme un problème public.}

Parallèlement, on assiste à une politisation accrue de la laïcité. Depuis 1989 et la première «affaire du voile» à Creil, la place de l'islam en France fait débat. Revenue au pouvoir en 2002, la droite entend réagir à ce qu'elle interprète comme une menace pour la laïcité. Trois évènements survenus en 2003 signent cette reprise en main : la création par Nicolas Sarkozy du Conseil français du culte musulman (CFCM) dans le but de structurer un «islam de France » apte à dialoguer avec les pouvoirs publics; la publication du rapport de François Baroin Pour une nouvelle laïcité, dans lequel le député RPR appelle de ses vœux une laïcité plus restrictive face à la montée de l'islamisme et du «communautarisme»; enfin, la mise en place par le président Jacques Chirac d'une commission de réflexion "sur l'application du principe de laïcité dans la République», qui débouchera sur l'adoption de la loi du 15 mars 2004 interdisant les signes religieux ostensibles à l'école.

Alors que l'État réaffirme fermement l'un des fondements de la République et célèbre le centenaire de la loi de séparation des Églises et de l'État, il développe une nouvelle rhétorique a priori peu compatible avec l'ethos républicain : celle de la promotion de la diversité. Directement importées des États-Unis, ces deux notions sont promues par des think tanks libéraux (Institut Montaigne et IMS-Entreprendre pour la Cité) qui y voient une façon de s'attaquer aux inégalités tout en évitant l'approche antidiscriminatoire jugée culpabilisante. Le gouvernement de Dominique de Villepin reprend à son compte ce référentiel en faisant adopter la loi pour l'égalité des chances du 31 mars 2006 et en promouvant la Charte de la diversité par l'intermédiaire d'Azouz Begag, ministre délégué à la Promotion de l'égalité des chances de 2005 à 2007.

Autre signe de cet infléchissement, le Fasild devient l'Agence nationale pour la cohésion sociale et l'égalité des chances (Acsé). Malgré ce changement de nom, l'établissement continue à promouvoir la lutte contre les discriminations en finançant des diagnostics, des formations et des plans de lutte territoriaux. De leur côté, les grandes entreprises signataires de la Charte de la diversité se dotent de plans d'action. Tout ceci génère une demande d'expertise qui se traduit par la création d'un «marché» et l'apparition de cabinets spécialisés.

En 2008, suite aux élections municipales remportées par la gauche, un grand nombre de

2. Edwin Hatton, Faire aimer la laïcité : la mobilisation des collectivités avant et après les attentats de 2015, Mémoire réalisée sous la direction de Gwénaële Calvès dans le cadre du diplôme universitaire "Laïcité et principes de la République », Cergy-Pontoise, Université de Cergy-Pontoise, 2017.

3. Mon panel était composé de dix-sept collectivités, dont douze municipalités (Aubervilliers, Bordeaux, Dijon, Évry, Grenoble, Lyon, Paris, Rennes, Rezé, Roubaix, Toulouse, Villeurbanne), deux intercommunalités (Brest et Grenoble), deux conseils départementaux (Haute-Garonne et Seine-Saint-Denis) et un conseil régional (Île-de-France)

4. Didier Fassin, "L'invention française de la discrimination », in Revue française de science politique, vol. 52, n 4, 2002, pp. 403-423 
municipalités créent des missions de lutte contre les discriminations. Au même moment, l'État donne un signal contraire en choisissant d'affaiblir la Halde, jugée trop critique et indépendante. Sa nouvelle présidente, Jeannette Bougrab, s'attache à mettre au pas l'institution en désavouant un avis rendu en mars dans l'affaire de la crèche Baby Loup. Alors que le service juridique de la Halde avait qualifié de discriminatoire le licenciement par une crèche d'une salariée pour avoir porté le voile, la nouvelle présidente de l'institution prend le parti de la crèche. Un an plus tard, la Haute Autorité est fusionnée dans le Défenseur des droits, ce qui entraîne une baisse de visibilité de la lutte contre les discriminations et une chute des saisines pour ce motif. La promotion de la diversité, elle, continue sa progression avec la création en 2008 du Label diversité, voulu par l'État et délégué à l'Afnor certification.

\section{L'émergence des politiques de laïcité dans le giron de la lutte contre les discriminations}

Dans les formations à la non-discrimination qui sont organisées à cette période, l'évocation de la religion suscite parfois des débats. Ils sont généralement centrés sur l'islam et plus précisément sur le voile. Les intermédiaires de l'emploi se demandent, par exemple, s'ils doivent conseiller aux femmes voilées de se découvrir pour trouver du travail. La religion n'est pas encore abordée comme une problématique à part entière mais comme une sous-thématique de la discrimination ou de la diversité. Ainsi, en mars 2009, l'association IMS-Entreprendre pour la Cité publie à l'intention de ses adhérents un guide pratique intitulé «Gérer la diversité religieuse en entreprise».

Dans les administrations, la prise en compte de cette question est encore balbutiante. La «Charte de la laïcité dans les services publics» diffusée par circulaire ministérielle le 13 avril 2007, entre les deux tours de l'élection présidentielle, est passée complètement inaperçue. Pourtant, les services publics se trouvent de plus en plus confrontés à des formes d'expression religieuse ou à des demandes d'accommodement qui les déstabilisent. En réponse à cette situation, quelques collectivités territoriales pionnières (Brest, Paris et Grenoble) se saisissent du sujet et organisent, entre 2009 et 2011, les premières formations sur la laïcité et la gestion du fait religieux.

Ces stages, qui rencontrent un véritable succès auprès des professionnels, relèvent d'initiatives des services municipaux ou intercommunaux et non d'une commande politique. Les élus éprouvent au contraire un certain malaise vis-à-vis de cette question jugée trop sensible. Il est vrai qu'en pleine présidence de Nicolas Sarkozy, l'islam s'affirme plus que jamais comme un point de clivage (polémique sur les prières de rue, "affaire Baby Loup», interdiction de la dissimulation du visage dans l'espace public, circulaire Chatel...). Prudents, les élus brestois, parisiens et grenoblois valident l'organisation de ces formations mais ne souhaitent pas leur donner trop de publicité. On observe la même discrétion à Toulouse où, après les attentats commis par Mohammed Merah en mars 2012, le maire décide de créer un Conseil de la laïcité réunissant des représentants des principaux cultes. Cette instance fonctionne d'abord "en catimini» avant d'être officialisée par une délibération du 10 décembre 2014 et rebaptisée «Toulouse fraternitéConseil de la laïcité».

\section{(c) En 2008, suite aux élections municipales remportées par la gauche, un grand nombre de municipalités créent des missions de lutte contre les discriminations. Au même moment, l'État donne un signal contraire en choisissant d'affaiblir la Halde, jugée trop critique et indépendante.}

De retour au pouvoir après les élections présidentielles et législatives de 2012, la gauche entend rompre avec les dérives nationalistes de la présidence de Nicolas Sarkozy. Le Premier ministre Jean-Marc Ayrault désactive le Haut Conseil à l'intégration $(\mathrm{HCl})$ et confie au conseiller d'État Thierry Tuot la rédaction d'un rapport sur la «refondation de la politique d'intégration». Celui-ci appelle à faire de la France une «société inclusive» en mettant notamment l'accent sur la lutte contre les discriminations. D'abord publiés dans une certaine discrétion, les documents en lien avec le rapport font ensuite l'objet d'une violente polémique après une série d'articles du Figaro qui leur reproche de vouloir faire le lit du «communautarisme», en prônant notamment la remise en question de la loi interdisant les signes religieux ostensibles à l'école ${ }^{5}$.

5. Fabrice Dhume, «L'intimidation : retour sur la campagne de presse qui a mené à l'enterrement des rapports », in Migrations Société, nº 155, 2014, pp. 131-152. 
Cet épisode, qui pousse le gouvernement à enterrer ces rapports, montre le caractère toujours inflammable de la question du voile à l'école, près de dix ans après la loi de 2004.

S'ils renoncent à refonder la politique d'intégration, le président de la République et son Premier ministre entendent afficher leur attachement à la laïcité face à ceux qui l'accusent de la trahir. Le 23 avril 2013, ils installent l'Observatoire de la laïcité, une instance créée formellement en 2007 par Jacques Chirac et Dominique de Villepin. Comme le $\mathrm{HCl}$, l'Observatoire de la laïcité est un organe consultatif placé sous l'autorité du Premier ministre mais il va s'attacher à promouvoir une tout autre vision de la laïcité, plus libérale et fidèle à la loi de 1905.

Malgré ce changement, le gouvernement poursuit l'œuvre de «pédagogie de la laïcité» prônée par le $\mathrm{HCl}$, en diffusant à la rentrée scolaire 2013 une «Charte de la laïcité à l'école». Sur la délicate question du port du voile par les parents accompagnant les sorties scolaires, le Conseil d'État rend le 23 décembre 2013 un avis stipulant que les parents d'élèves sont de simples usagers du service public et qu'ils ne sont donc pas soumis à l'obligation de neutralité. En octobre 2014, la nouvelle ministre de l'Éducation nationale Najat Vallaud-Belkacem confirme cette doctrine en déclarant que l'autorisation doit être la règle et l'interdiction l'exception.

Si elles sont désormais traitées avec plus de souplesse par le gouvernement, les questions de religion et de laïcité font toujours couler beaucoup d'encre. Ainsi, la Fondation Jean Jaurès publie un Guide pratique de la laïcité en deux volumes à destination des élus (2012), le Conseil économique, social et environnemental (CESE) consacre un rapport au «fait religieux dans l'entreprise» (2013) et le Conseil d'État édicte une note sur «Le juge administratif et l'expression des convictions religieuses» (2014).

\section{Le risque de la stigmatisation des personnes de culture musulmane}

Dans la fonction publique territoriale, tous les chargés de mission «Lutte contre les discriminations» attestent d'une montée en puissance des problématiques liées à la religion mais certains sont réticents à l'idée d'en faire une problématique à part entière. Ils craignent que cela ne contribue à renforcer la stigmatisation des personnes de culture musulmane et pointent le risque que la lutte contre les discriminations se retrouve prise en étau entre la diversité et la laïcité. Signes avant-coureurs de ce glissement, la mairie de Toulouse ouvre en février 2012 un «Espace des diversités et de la laïcité » et la mairie de Roubaix créé en 2013 un poste de chef de mission «laïcité, diversité et vivre-ensemble».

\section{(l. Comme le HCI, l'Observatoire de la laïcité est un organe consultatif placé sous l'autorité du Premier ministre mais il va s'attacher à promouvoir une tout autre vision de la laïcité, plus libérale et fidèle à la loi de 1905.}

Au niveau de l'État, si la lutte contre les discriminations n'est pas remise en cause, elle demeure cantonnée à la politique de la ville. La loi du 21 février 2014 maintient cet arrimage et fait de la lutte contre les discriminations une des «thématiques transversales» des nouveaux contrats de ville. Les collectivités sont invitées à la traiter en suivant une "approche intégrée» et en s'appuyant sur les instruments habituels de la lutte contre les discriminations (diagnostics, formations et plans territoriaux). Seul changement, ces dispositifs sont désormais gérés par le Commissariat général à l'égalité des territoires (CGET) qui a absorbé l'Acsé en mai 2014.

Le manque d'ambition est patent. Après la bataille du «mariage pour tous», le Président François Hollande ne souhaite pas initier de nouvelles avancées sociétales. La lutte contre les discriminations se voit également affaiblie par le retour au pouvoir de la droite dans de nombreuses villes suite aux élections municipales de mars. Cette alternance entraîne une baisse générale des moyens alloués à cette cause, comme à Amiens où la métropole supprime sa Maison de l'égalité.

\section{De la célébration de la laïcité à l'effacement de la lutte contre les discriminations}

Les attentats de janvier 2015 intensifient la mobilisation des pouvoirs publics en faveur de la laïcité. Interprétés comme une attaque contre les valeurs républicaines, ils suscitent une réponse immédiate du gouvernement qui lance le 22 janvier une «grande 
mobilisation de l'école pour les valeurs de la République», dont la laïcité constitue une priorité. Le 6 mars, le premier Comité interministériel à l'égalité et à la citoyenneté ( $\mathrm{Ciec}$ ) annonce un ensemble de mesures destinées à promouvoir la laïcité, dont un vaste plan de formation national intitulé «Valeurs de la République et laïcité » et destiné à former dix mille personnes par an.

Dans les mois qui suivent, toutes les grandes administrations se saisissent du sujet en formant leur personnel et en publiant des guides pratiques sur la laïcité. Un «benchmark ${ }^{6}$ » commandé en 2016 par la ville de Lyon n'en recense pas moins de vingt-cinq, secteurs public et privé confondus. S'appuyant sur un diagnostic préalable, ces guides répertorient les situations problématiques les plus courantes et indiquent la façon de les traiter. Des groupes de travail internes ou des hotlines sont parfois créés pour recueillir et analyser les signalements, comme à Roubaix ou à Lyon. Ainsi, les attentats obligent les collectivités à s'intéresser à des situations de tension qui bien souvent existaient avant ces événements mais font désormais l'objet d'une attention particulière.

Appliquer et expliquer la laïcité ne suffit pas, il faut aussi la célébrer. Le 9 décembre 2015, jour du cent-dixième anniversaire de la loi de séparation des Églises et de l'État, l'Observatoire de la laïcité organise la première "Journée de la laïcité» au cours de laquelle est remis le premier «Prix de la laïcité de la République française». Dès l'année suivante, des collectivités territoriales (Bordeaux, Rennes, Rezé, Conseil départemental de Haute-Garonne...) célèbrent à leur tour cette date anniversaire à travers différentes manifestations: conférences, projections, spectacles, plantation d'un "arbre de la laïcité», visites de lieux de culte, rencontres interreligieuses...

À travers toutes ces actions, les collectivités espèrent fortifier l'attachement de leurs administrés au principe de laïcité, tout en favorisant la connaissance de l'Autre, le dialogue interreligieux et le «vivre-ensemble». Certaines vont plus loin en créant des instances de dialogue et de concertation dédiées à la laïcité: Conseil inter religieux et citoyen de Bordeaux (2008), Observatoire parisien de la laïcité (2012), Conseil de la laïcité de Toulouse (2013), Comité consultatif laïcité de Rennes (2015), Conseil citoyen local de la laïcité d'Évry (2016)... En invitant des représentants religieux, des élus, des acteurs associatifs et des experts de tous bords à dialoguer, les collectivités se posent en gardiennes de la laïcité et en organisatrices du débat citoyen sur le sujet.

Que devient la lutte contre les discriminations dans cette avalanche d'actions en faveur de la laïcité? Comme nous l'avons déjà souligné, ce sont souvent les fonctionnaires ou les services en charge de l'égalité et/ou de la diversité qui se retrouvent chargés de la laïcité. Ainsi, au CGET, le lancement du plan «Valeurs de la République et laïcité» a été confié à des fonctionnaires qui étaient en charge des dispositifs de lutte contre les discriminations au Fasild puis à l'Acsé. De même, sur les dix-sept collectivités territoriales que nous avons examinées, dix ont fait le choix de confier à leur service "égalité » les actions relatives à la laïcitér. La ville d'Évry, qui ne disposait pas d'un tel service, a créé en 2016 un poste de chargé de mission Laïcité-égalité femmes-hommes. La mairie de Paris a également ouvert un poste de chargé de mission Citoyenneté-laïcité et l'a confié à l'ancienne responsable de la Mission égalité femmes-hommes. À Rennes, les relations avec les cultes et la laïcité ont été gérées par la Mission égalité jusqu'en 2014, puis transférées à une chargée de mission rattachée à la direction Action territoriale tranquillité publique, afin de «ne pas réduire la religion à la discrimination». De même, d'autres collectivités dotées d'une mission égalité ont pris le parti de confier la laïcité à un autre service : direction du personnel (Conseil départemental de Seine-Saint-Denis), Mission médiation (Roubaix) ou cabinet du président (Conseil départemental de Haute-Garonne).

\section{(6) Au niveau de l'État, si la lutte contre les discriminations n'est pas remise en cause, elle demeure cantonnée à la politique de la ville.}

La porosité entre lutte contre les discriminations et promotion de la laïcité se manifeste également dans les contenus des formations ou des groupes de travail, qui allient souvent les deux thématiques, comme à Aubervilliers, à Brest, à Grenoble, à Lyon, à Rezé ou à Roubaix. Même lorsque la laïcité et l'égalité sont portées par des services différents, la

\footnotetext{
6. Ludovic Vievard, Laïcité : benchmark des guides et des démarches sur la laïcité, Lyon, Grand Lyon prospective, juin 2016 7. Aubervilliers, Bordeaux, Brest Métropole Océane, Dijon, Évry, Grenoble et Grenoble-Alpes Métropole, Lyon, Toulouse, Villeurbanne et le Conseil régional d'Île-de-France.
} 
coopération est la règle, comme à Rennes où la référente laïcité participe au Plan de lutte contre les discriminations. Au Conseil départemental de HauteGaronne, l'élue en charge de l'égalité femmes-hommes siège au comité de sélection des actions du parcours laïque et citoyen. On n'observe donc pas de concurrence entre ces deux causes, même si la laïcité tend à prendre le dessus en raison de la médiatisation dont elle fait l'objet.

\section{Inflation des normes et inertie politique}

Le lien entre laïcité et lutte contre les discriminations est aussi perceptible dans les discours politiques. Dans l'éditorial de son guide pratique sur la laïcité, la maire de Paris revendique une «laïcité d'intégration et non d'exclusion, d'ouverture et non de fermeture [...]. C'est à cette condition que notre précieux service public tiendra sa promesse d'égalité et restera fidèle à ses valeurs ${ }^{8}{ }^{\prime}$. L'égalité est aussi mise en avant par le président du Conseil départemental de HauteGaronne, qui place en exergue de son éditorial au Parcours laïque et citoyen cette citation de Jean Jaurès: "Assez parlé d'égalité, il est temps de faire des égaux ${ }^{9} 》$. Certains élus dénoncent également l'instrumentalisation de la laïcité. Adjoint au maire de Bordeaux en charge de l'égalité et de la citoyenneté, Marik Fetouh dénonce la «laïcité identitaire» prônée par l'extrême droite: "Cette laïcité, dirigée contre une religion [l'islam], ne fait qu'augmenter la stigmatisation et la discrimination ressenties et favorise donc au final le phénomène qu'elle prétend combattre ${ }^{10}$.»
La loi du 20 avril 2016 relative à la déontologie et aux droits et obligations des fonctionnaires consacre la neutralité confessionnelle des agents publics et la loi du 8 août 2016, dite "Loi Travail», introduit la possibilité pour les entreprises d'inscrire dans leur règlement intérieur des restrictions à la liberté de manifester sa religion (interdiction du port de signes religieux, notamment). La présidence Hollande se caractérise donc par une mobilisation sans précédent des pouvoirs publics en faveur de la laïcité mais sans modification substantielle de ses contours. La vision qui domine l'action gouvernementale est qu'il faut faire connaître le droit existant plutôt que le renforcer.

En matière de lutte contre les discriminations, on constate en revanche une inflation de nouvelles normes avec l'intégration de critères de discrimination toujours plus spécifiques ${ }^{11}$, la mise en place de l'action de groupe en matière de discrimination au travail et l'obligation de formation des recruteurs à la non-discrimination dans les entreprises de plus de 300 salariés. Ces avancées sont intéressantes mais, faute de réflexion sur leur application, elles risquent de demeurer ineffectives. Cette fuite en avant législative cache mal l'impuissance des pouvoirs publics à faire reculer les discriminations. Pourtant, une étude de France stratégie réalisée en 2016 a évalué que la suppression des discriminations à l'emploi sur les seuls critères du sexe et de l'origine pourrait générer une hausse du produit intérieur brut (PIB) de 3,6 à $14 \%{ }^{12}$.

8. Secrétariat général de la Mairie de Paris, Laïcité et neutralité au sein des services publics de la Ville de Paris : guide pratique à l'usage des encadrant-e-s, éditorial d'Anne Hidalgo, décembre 2015, p. 3.

9. Le parcours laïque et citoyen au collège, 2016-2017, éditorial de Georges Méric, mai 2016, p. 4.

10. Marik Fetouh, «La ville de Bordeaux et les religions : la laïcité au service du vivre-ensemble», in Charles Mercier, Jean-Philippe Warren (dir), Identités religieuses et cohésion sociale. La France et le Québec à l'école de la diversité, Lormont, Le bord de l'eau éd., 2016, p. 303.

11. L'identité sexuelle (2012), le lieu de résidence (2014), « la particulière vulnérabilité résultant de la situation économique apparente ou connue » (2016), la perte d'autonomie, la domiciliation bancaire, « la capacité à s'exprimer dans une langue autre que le français » (2017), la discrimination commise pour «avoir subi ou refusé de subir » du harcèlement sexuel (2012) ou des faits de bizutage (2017).

12. France Stratégie, « Le coût économique des discriminations », rapport à la ministre du Travail, de l'Emploi, de la Formation professionnelle et du Dialogue social, et au ministre de la Ville, de la Jeunesse et des Sports, septembre 2016. 


\section{Conclusion}

Vingt ans après la reconnaissance publique des discriminations en France, nous nous trouvons face à un paradoxe : alors que ce phénomène n'a jamais été aussi documenté, il s'est progressivement effacé de l'agenda politique ${ }^{13}$, ce qui fait dire à Patrick Simon que «la lutte contre les discriminations n'a pas eu lieu $^{14} »$. Cette démobilisation des pouvoirs publics n'est peut-être pas étrangère à la radicalisation d'une partie du mouvement antiraciste. Aujourd'hui, la lutte

\section{(2) Au niveau national, la fin du quinquennat} de François Hollande est marquée par des tensions entre le Premier ministre, Manuel Valls, tenant d'une laïcité de combat, et l'Observatoire de la laïcité partisan d'une laïcité inclusive.

contre les discriminations n'a pas complètement disparu mais elle occupe une place marginale dans l'action publique. Certaines collectivités poursuivent leur action dans ce domaine en créant, par exemple, des «réseaux de vigilance» (Villeurbanne, Paris, Aubervilliers, Grenoble...), tandis que l'État semble se contenter de promouvoir le Label diversité et de financer des testings. A contrario, la pédagogie de la laïcité s'est imposée comme une véritable politique publique et a bénéficié de moyens importants, ce qui a permis aux acteurs publics d'être mieux outillés pour prévenir et traiter les atteintes à la laïcité.
Sans minorer l'importance de la laïcité, on peut s'interroger sur la prééminence qui lui a été donnée ces dernières années. Cette survalorisation ne risquet-elle pas de conférer à la religion une place disproportionnée dans un pays où deux-tiers des personnes se déclarent athées ou sans religion ${ }^{15}$ ? Certes, la France connaît, comme le reste du monde, un retour du religieux et la question du "vivreensemble» s'y pose de façon peut-être plus aigüe qu'ailleurs étant donnée la grande diversité de sa population. Cependant, faire de la laïcité la seule réponse à cette question est insuffisant. La laïcité n'est pas une fin en soi mais un principe visant à garantir la liberté de religion et l'égalité entre tous les citoyens, quelles que soient leurs convictions. Elle ne permet pas de lutter contre les discriminations en raison du sexe, de l'origine, de l'âge ou du handicap, ni de réduire les inégalités sociales, autant de maux qui minent davantage le «vivre-ensemble» que les atteintes à la laïcité. Il serait donc temps de remettre la laïcité à sa juste place et de rouvrir le chantier de l'égalité.

13. Fabrice Dhume, «De la reconnaissance à l'effacement: la politique française de lutte contre les discriminations et la question raciale », in Félix Boggio Ewanje-Épée, Stella MaglianiBelkacem (dir.), Race et capitalisme, Paris, éd. Syllepse, coll. "Arguments et mouvements », 2012, pp. 51-66.

14. Patrick Simon, «La lutte contre les discriminations n'a pas eu lieu : la France multiculturelle et ses adversaires », in Mouvements, $n^{\circ} 83,2015$, pp. 87-95.

15. Selon un sondage WIN-Gallup International réalisé en 2012 , 34 \% des Français se déclarent « non-religieux » et 29 \% « athées convaincus », contre seulement $37 \%$ de « religieux». 